\title{
RELACIÓN ENTRE INTELIGENCIA ESPIRITUAL Y SATISFACCIÓN EXISTENCIAL CON LA EXPERIENCIA PARANORMAL
}

\author{
Alejandro PARRA \\ http://orcid.org/0000-0001-7943-2794 \\ NATALIA RIBILLA \\ Facultad de Psicología y Relaciones Humanas \\ Universidad Abierta Interamericana, Buenos Aires, Argentina \\ Correo electrónico: rapp_ale@fibertel.com.ar \\ Recibido: 11 de septiembre del 2020 / Aceptado: 7 de octubre del 2020 \\ doi: https://doi.org/10.26439/persona2020.n023(2).4854
}

\begin{abstract}
Resumen. El objetivo de este estudio es evaluar la frecuencia de experiencias paranormales y su relación con el grado de inteligencia espiritual y el nivel de satisfacción existencial. Una muestra integrada por 200 participantes de ambos sexos completó tres instrumentos: el Cuestionario de Experiencias Paranormales, el Cuestionario de Inteligencia Espiritual y la Escala de Satisfacción Existencial. Los resultados muestran que los encuestados indicaron haber tenido al menos un tipo de estas experiencias, y que la inteligencia espiritual es un fuerte predictor; la relación con la satisfacción existencial resultó también estadísticamente significativa. Estas experiencias producen un sentimiento de satisfacción espiritual, lo que sugiere que pueden inducir efectos positivos y -lejos de resultar perturbadoras - contribuyen a la capacidad del individuo para resolver cuestiones de significado y de valor existencial. Concluimos que la inteligencia espiritual podría ser más adaptativa para aquellos que buscan un significado a sus experiencias paranormales, esto es, para aquellas personas que practican yoga, meditación, recuerdan sueños o tienen una vida mental más rica en fantasía, y quienes comparten un contexto más amplio de significado frente a experiencias que acompañan su estilo de vida espiritual. Una forma de "evidencia" que confirma el sentido de sus prácticas religiosas o espirituales, que pueden ser vistas como un indicador que moldea o reafirma la congruencia entre sus creencias espirituales.
\end{abstract}

Palabras clave: experiencias paranormales / inteligencia espiritual / satisfacción existencial / espiritualidad / psicología positiva 


\title{
RELATIONSHIP BETWEEN SPIRITUAL INTELLIGENCE AND EXISTENTIAL SATISFACTION WITH PARANORMAL EXPERIENCE
}

\begin{abstract}
The aim of this study is to assess the frequency of paranormal experiences and their relationship with the levels of spiritual intelligence and existential satisfaction. Three instruments were administered to a sample of 200 male and female participants: the Paranormal Experiences Survey, the Spiritual Intelligence SelfReport Inventory and the Existential Satisfaction Scale. The results show that survey respondents had at least one of these experiences, and that spiritual intelligence is a strong predictor of them. The relationship with existential satisfaction was also significant. These experiences result in a feeling of spiritual satisfaction, thus suggesting that they can generate positive effects and-far from being disturbing-contribute to the individual's ability to solve meaning and existential value issues. In conclusion, spiritual intelligence could be more adaptive for those who seek a meaning for their paranormal experiences, i.e., for those people who practice yoga or meditation, recall dreams, have a mental life richer in fantasy, and share a broader context of meaning in the face of the experiences of their spiritual lifestyle: a form of "evidence" that confirms the sense of their religious or spiritual practices, and that can be seen as an indicator that shapes or reaffirms the consistency between their spiritual practices.
\end{abstract}

Keywords: paranormal experiences / spiritual intelligence / existential satisfaction / spirituality / positive psychology 


\section{INTRODUCCIÓN}

Zohar (1997) acuñó el término inteligencia espiritual e introdujo la idea de que es la inteligencia que nos hace completos, que nos da nuestra integridad... Es la inteligencia del alma, la inteligencia del yo profundo. Es la inteligencia con la que hacemos preguntas fundamentales y con la que replanteamos nuestras respuestas. Según Emmons (1999), “la inteligencia espiritual es un marco para identificar y organizar las destrezas y habilidades necesarias para el uso adaptativo de la espiritualidad" (p. 17) y propuso inicialmente cinco componentes: (a) la capacidad para utilizar recursos espirituales para resolver problemas; (b) la capacidad para entrar en estados elevados de conciencia; (c) la capacidad para invertir experiencias cotidianas; (d) la capacidad de trascendencia de lo físico y material; y (e) la capacidad para ser virtuoso (Emmons, 2000b).

Varios autores (Vaughan, 2002; Wigglesworth, 2006; Wolman, 2001) sugieren que la inteligencia espiritual surge a medida que la conciencia evoluciona hacia una forma cada vez más profunda de la materia, la vida, el cuerpo, la mente, el alma y el espíritu. Esta parece conectar lo personal con lo transpersonal y el yo con el espíritu. La inteligencia espiritual va más allá del desarrollo psicológico convencional. Además de la autoconciencia, implica la conciencia de nuestra relación con lo trascendente, entre nosotros, con la tierra y con todos los seres (Srivastava, 2014).

Tradicionalmente, el foco principal de estudio en psicología se concentra en la psicopatología y el alivio de los síntomas (Barlow y Durand, 2005), mientras que el estudio del bienestar, la espiritualidad y las emociones positivas en la población clínica y no clínica se ha dejado de lado (Paloutzian y Ellison, 1982; Sheldon y King, 2001; Seligman y Csikszentmihalyi, 2000). Sin embargo, en el campo de la psicología positiva se ha realizado un esfuerzo para diseñar, implementar y evaluar intervenciones para promover el bienestar y la espiritualidad (Sin y Lyubomirsky, 2009).

En investigaciones recientes se ha observado el interés de muchas personas por una visión abierta a aspectos de la vida que van más allá del reino de lo físico y de lo material, y que pueden conducir a la salud y al bienestar (Baer, 2003; Bishop et al., 2004; Hölzel et al., 2011). Un aspecto destacable de las experiencias espirituales/paranormales es que, aunque son singulares y transitorias, tienen un enorme impacto en las personas que las experimentan. Un individuo puede tener un cambio en valores después de una experiencia cercana a la muerte o una experiencia de sanación espiritual (Greyson, 1996). Según Noble (2000) y Vaughan (2002), las experiencias espirituales contribuyen al desarrollo individual de la inteligencia espiritual (ver, por ejemplo, Emmons 2000a; Holmes, Solomon, Cappo y Greenberg, 1993; Lehmann, Faber, Achermann, Jeanmonod y Gianotti, 2001; Love, 2002; Piechowski, 2001), lo que sugiere que esta aumenta con la edad y las situaciones vividas. 
Sin embargo, las experiencias paranormales también pueden promover la salud, pero existe muy poca investigación en psicología sobre sus efectos en la vida de las personas (Cardeña, Krippner y Lynn, 2014). Por otro lado, se ha discutido el hecho de que algunas personas se sientan perturbadas por estas experiencias y que necesiten ayuda (Gómez Montanelli y Parra, 2004, 2005). En efecto, quienes están interesados en temas paranormales y han tenido experiencias que interpretan como tales indicaron que estas habían reforzado sus creencias espirituales y su percepción subjetiva de bienestar. El miedo se presenta como una de las reacciones iniciales más comunes frente a un fenómeno paranormal, pero los efectos a largo plazo parecen ser positivos (Kennedy, Kanthamani y Palmer 1994; Kennedy y Kanthamani, 1995; Parra, 2008). Otras investigaciones han sugerido que la capacidad de construir significado es más adaptativa (ver Mascaro y Rosen, 2005).

El objetivo general de este estudio es evaluar la frecuencia de experiencias paranormales y su relación con el grado de inteligencia espiritual y el nivel de satisfacción existencial, más específicamente: relacionar la frecuencia de experiencias paranormales con la inteligencia espiritual y la satisfacción existencial. Se hipotetiza que se encontrará (H1) una correlación positiva y significativa entre la frecuencia de experiencias paranormales y la inteligencia espiritual, y $(\mathrm{H} 2)$ una correlación positiva y significativa entre la frecuencia de experiencias paranormales y la satisfacción existencial.

\section{MÉTODO}

\section{Participantes}

Se reunió una muestra no probabilística integrada por 200 casos, 48 varones (24\%) y 152 mujeres (76 \%), cuyo rango etario era de 18 a 75 años (media $=40,04$, DT $=12,94$ ). Respecto a las características demográficas, casi la mitad era profesional (45\%), y se halló una distribución de empleados (11\%), desempleados (16\%) y comerciantes (10\%), con ingresos aceptables para vivir (75\%), casi la mitad de religión católica (45 \%) y convivientes en pareja (44\%).

\section{Instrumentos}

Cuestionario de Experiencias Paranormales (CEP; Parra y Argibay, 2013a, 2013b; Parra y Corbetta, 2013). Es un cuestionario autoadministrable diseñado para recoger información sobre tipo y frecuencia de experiencias paranormales espontáneas, que incluyen sueños premonitorios (imágenes oníricas que anticipan el futuro), percepción extrasensorial (telepatía, clarividencia y precognición), ver el aura (percepción visual del campo energético), experiencia fuera del cuerpo (autopercepción del yo localizado en otra parte fuera del cuerpo físico), experiencia mística (vivencia mental extraordinaria 
bajo un estado no ordinario de conciencia), sanación anómala (experiencia de sanar a otra persona a distancia) y oír voces/ver apariciones (percepción auditiva o visual de una entidad no física). Cada ítem tiene una escala de Likert donde $0=$ nunca, $1=$ una vez, $2=$ múltiples veces. La consistencia interna de este inventario es buena, con un coeficiente de confiabilidad alfa de Cronbach de .93, y confiabilidad test-retest también aceptable. Se empleó un índice de experiencias (Index Psi) para cada caso en función de las respuestas a cada experiencia reportada, con un rango de respuesta de 0 a 24 (siendo 24 la puntuación más alta).

Cuestionario de Inteligencia Espiritual (SISRI-24; King y DeCicco, 2009; King, Mara y DeCicco, 2012). Mide la capacidad para tratar y resolver cuestiones de significado y valor, es decir, adaptar el comportamiento y la vida en el contexto más amplio del significado. Está compuesto por 24 ítems divididos en cuatro componentes:

1. Pensamiento crítico existencial: consideración del propósito o existencia de una persona y la relación con el universo, así como temas de la vida, la muerte, la realidad, la verdad o la justicia (por ejemplo: "He meditado bastante acerca del propósito o la razón de mi existencia"; "A veces pienso profundamente acerca de lo que sucede más allá de la muerte").

2. Producción de significado personal: la capacidad de crear significado y propósito en la propia vida, tanto en la experiencia mental y física, e incluso en el fracaso (por ejemplo: "Tener la habilidad para encontrar el significado y el propósito en la vida me ayuda a adaptarme a situaciones estresantes"; "Soy capaz de definir un propósito, o una razón, para mi vida").

3. Conciencia trascendental: la capacidad de ver una imagen más grande que lo meramente físico y más allá de la experiencia habitual. Conciencia de la existencia de una dimensión espiritual que puede verse en otros y observarse personalmente (por ejemplo: "Estoy consciente de la existencia de una profunda conexión con el otro"; "Me defino como un ser trascendente y no físico").

4. Expansión del estado consciente: el poder de controlar y pasar a un nivel superior o espacio espiritual a través de la meditación profunda, oración o consideración (por ejemplo: "Tengo la habilidad de ingresar a otros estados no ordinarios de conciencia"; "Puedo controlarme cuando ingreso a estados superiores de conciencia").

La escala cuenta con varias versiones en diferentes idiomas y fue validada por expertos y sometida a un análisis de confiabilidad mediante el uso del alfa de Cronbach, con buenos resultados de consistencia interna $(\alpha=.85)$. 
Escala de Satisfacción Existencial (Boado de Landaboure, 2001; Längle, 2004). Evalúa las competencias personales y existenciales para poder tratar consigo mismo(a) y con el mundo. Consta de 46 ítems con una puntuación que va desde 1 (absolutamente en desacuerdo) a 6 (absolutamente de acuerdo). El índice de confiabilidad medido con el alfa de Cronbach es de .83 para esta muestra ( $N=9$ ítems). Contiene cuatro subescalas, las primeras dos miden la función personal y las dos últimas, la función existencial:

1. Autodistanciamiento: capacidad para organizar el espacio libre interior. Se pone de manifiesto en la posibilidad de conquistar la distancia de sí mismo, deseos, representaciones, temores, motivos, permitiendo la libre captación del mundo aun en momentos difíciles de la vida.

2. Autotrascendencia: capacidad del hombre para percibir y aceptar al otro de acuerdo con sus valores, lo que permite el compromiso con el mundo. Esta subescala evalúa la capacidad de percibir (sensibilizarse frente a) los valores, lo que se pone de manifiesto en la claridad de los sentimientos.

3. Libertad: capacidad de tomar decisiones y elegir libremente. Evalúa la capacidad de decisión que tiene la persona al encontrarse ante una posibilidad real de acción, acorde con una jerarquía valorativa. Cuando en repetidas ocasiones ha logrado elegir sin mayores problemas, surge el sentimiento de ser libre y permite ver tanto la capacidad o incapacidad de decisión como también si la persona es capaz de tomar "decisiones seguras".

4. Responsabilidad: implica hacerse responsable de las consecuencias de los propios actos. El objetivo de esta subescala es evaluar la responsabilidad como disposición para comprometerse a partir de una decisión libre y siendo consciente de la obligación, así como de las tareas y valores que dicha decisión implica.

\section{Procedimiento}

Se distribuyeron los instrumentos en un sobre cerrado, entregado en mano, y se dieron instrucciones para completarlos. Los datos fueron tratados con confidencialidad y anonimato de las respuestas. Fueron excluidos los participantes que presentaron los siguientes indicadores: (1) trastorno neurológico o traumatismo que resulte en déficit cognitivo; (2) uso recreativo de drogas o alcohol con criterio de consumo de riesgo; (3) cuestionarios respondidos en forma incorrecta, con tachaduras o enmiendas, o incompletos. Los participantes firmaron un formulario de consentimiento y se les informó que podían negarse a participar. La participación fue voluntaria y sin pago. 


\section{RESULTADOS}

Se llevó a cabo un contraste de hipótesis sobre la normalidad de las variables mediante un análisis de Kolmogorov-Smirnov. A partir de los valores obtenidos, se asumió una distribución asimétrica de las puntuaciones de los tres instrumentos. En consecuencia, se decidió emplear para los análisis estadísticos el test $H$ de Kruskal-Wallis o $U$ de MannWhitney para comparar grupos, y el coeficiente rho de Spearman para correlacionar las puntuaciones de las escalas.

La experiencia paranormal más comúnmente indicada fue sensación de presencia (75,5\%), seguida de experiencia mística (64\%), sueños premonitorios (63\%) y percepción extrasensorial (60\%), como se observa en la tabla 1.

Tabla 1

Frecuencia de experiencias paranormales*

\begin{tabular}{lll}
\hline Experiencia paranormal & $N$ & $\%$ \\
\hline Sensación de presencia & 151 & 75,5 \\
Experiencia mística & 128 & 64,0 \\
Sueños premonitorios & 126 & 63,0 \\
Percepción extrasensorial & 121 & 60,5 \\
Oír voces/ver apariciones & 102 & 51,0 \\
Experiencia fuera del cuerpo & 92 & 46,0 \\
Sanar a distancia & 89 & 44,5 \\
Ver el aura & 70 & 35,0 \\
\hline
\end{tabular}

* Ordenadas de mayor a menor

Elaboración propia

La H1 predice que se encontrará una correlación positiva y significativa entre la frecuencia de experiencias paranormales y la inteligencia espiritual. Esto se confirmó $\left(r_{s}=.62 ;\right.$ Sig. $\left.=.001\right)$, y se replicó para todas las experiencias paranormales (media $r_{s}=47$; $<.001)$ y los cuatro factores de inteligencia espiritual (media $\left.r_{s}=68 ;<.001\right)$ (ver la tabla 2). 
Tabla 2

Correlación entre frecuencia de experiencias paranormales e inteligencia espiritual

\begin{tabular}{|c|c|c|c|c|c|}
\hline Experiencias paranormales & $\begin{array}{c}\text { 1. Pensamiento } \\
\text { crítico } \\
\text { existencial }\end{array}$ & $\begin{array}{l}\text { 2. Producción } \\
\text { de significado } \\
\text { personal }\end{array}$ & $\begin{array}{l}\text { 3.Conciencia } \\
\text { trascendental }\end{array}$ & $\begin{array}{l}\text { 4. Expansión } \\
\text { de estado } \\
\text { consciente }\end{array}$ & $\begin{array}{c}\text { Inteligencia } \\
\text { espiritual }\end{array}$ \\
\hline 1. Sueños premonitorios & $.16^{* *}$ & $.21^{* * *}$ & $.32^{* * *}$ & $.37^{* * *}$ & $.31^{* * *}$ \\
\hline 2. Percepción extrasensorial & $.35^{* * *}$ & $.37^{* * *}$ & $.43^{* * *}$ & $.50^{* * *}$ & $.47^{* * *}$ \\
\hline 3. Ver el aura & $.34^{* * *}$ & $.35^{* * *}$ & $.42^{* * *}$ & $.53^{* * *}$ & $.47^{* * *}$ \\
\hline 4. $\mathrm{EFC}^{(1)}$ & $.31^{* * *}$ & $.23^{* * *}$ & $.40^{* * *}$ & $.47^{* * *}$ & $.41^{* * *}$ \\
\hline 5. Experiencia mística & $.44^{* * *}$ & $.37^{* * *}$ & $.53^{* * *}$ & $.50^{* * *}$ & $.54^{* * *}$ \\
\hline 6. Sensación de presencia & $.39^{* * *}$ & $.20^{* *}$ & $.40^{* * *}$ & $.35^{* * *}$ & $.41^{* * *}$ \\
\hline 7. Sanar a distancia & $.435^{* * *}$ & $.34^{* * *}$ & $.53^{* * *}$ & $.53^{* * *}$ & $.53^{* * *}$ \\
\hline $\begin{array}{l}\text { 9. Oír voces/ver apariciones } \\
\text { Index Psi }\end{array}$ & $\begin{array}{l}.25^{* * *} \\
.48^{* * *}\end{array}$ & $\begin{array}{l}.16^{*} \\
.39^{* * *} \\
\end{array}$ & $\begin{array}{l}.33^{* * *} \\
.60^{* * *}\end{array}$ & $\begin{array}{l}.40^{* * *} \\
.63^{* * *}\end{array}$ & $\begin{array}{l}.34^{* * *} \\
.62^{* * *}\end{array}$ \\
\hline
\end{tabular}

${ }^{*} p<.05 ;{ }^{* *} p<.005 ;{ }^{* * *} p<.001$

(1) Experiencia fuera del cuerpo

Elaboración propoia

La H2 predice que se encontrará una correlación positiva y significativa entre la frecuencia de experiencias paranormales y la satisfacción existencial, lo cual se confirmó $\left(r_{s}=.14\right.$; Sig. $\left.=.02\right)$. Esta significación se replicó para sueños premonitorios $\left(r_{s}=.22\right.$; Sig. =.001), percepción extrasensorial $\left(r_{s}=.14\right.$; Sig. =.01), sanar a distancia $\left(r_{s}=.27\right.$; Sig.<.001) y para uno de los factores de satisfacción existencial (autotrascendencia $r_{s}=.15$; Sig. $=.01$ ) (ver la tabla 3).

Tabla 3

Correlación entre frecuencia de experiencias paranormales y satisfacción existencial

\begin{tabular}{|c|c|c|c|c|c|}
\hline & $\begin{array}{c}\text { 1. Auto- } \\
\text { distanciamiento }\end{array}$ & $\begin{array}{c}\text { 2. Auto- } \\
\text { trascendencia }\end{array}$ & $\begin{array}{c}3 . \\
\text { Libertad }\end{array}$ & $\begin{array}{c}4 . \\
\text { Responsabilidad }\end{array}$ & $\begin{array}{l}\text { Satisfacción } \\
\text { existencial }\end{array}$ \\
\hline $\begin{array}{l}\text { 1. Sueños } \\
\text { premonitorios }\end{array}$ & .06 & $.19^{* *}$ & $.22^{* * *}$ & $.20^{* *}$ & $.22^{* * *}$ \\
\hline $\begin{array}{l}\text { 2. Percepción } \\
\text { extrasensorial }\end{array}$ & .07 & $.16^{* *}$ & .12 & .10 & $.14^{* *}$ \\
\hline 3. Ver el aura & .04 & $.14^{*}$ & .06 & .11 & .12 \\
\hline 4. $\mathrm{EFC}^{(1)}$ & .10 & $.12^{*}$ & .07 & .05 & .11 \\
\hline $\begin{array}{l}\text { 5. Experiencia } \\
\text { mística }\end{array}$ & .05 & .04 & .02 & .04 & .05 \\
\hline $\begin{array}{l}\text { 6. Sensación } \\
\text { de presencia }\end{array}$ & .03 & .02 & -.01 & -.02 & .01 \\
\hline $\begin{array}{l}\text { 7. Sanar a } \\
\text { distancia }\end{array}$ & $.19^{* *}$ & $.23^{* * *}$ & $.21^{* * *}$ & $.24^{* * *}$ & $.27^{* * *}$ \\
\hline $\begin{array}{l}\text { 9. Oír voces/ver } \\
\text { apariciones }\end{array}$ & -.01 & .08 & .04 & .02 & .05 \\
\hline Index Psi & .08 & $.15^{* *}$ & .09 & .09 & $.14^{* *}$ \\
\hline
\end{tabular}

${ }^{*} p<.05 ;{ }^{* *} p<.005 ;{ }^{* * *} p<.001$

(1) Experiencia fuera del cuerpo

Elaboración propia 
Se llevó a cabo un análisis de las principales características demográficas de la muestra, como género, ingresos económicos, espiritualidad y edad, religión y estado marital. Aunque no se encontraron diferencias de género, sin embargo, síse hallaron correlaciones positivas y significativas entre Index Psi con espiritualidad $\left(r_{s}=.46\right.$; Sig. <.001), satisfacción existencial con ingresos $\left(r_{s}=.14\right.$; Sig. $\left.=.02\right)$, espiritualidad $\left(r_{s}=.20 ;\right.$ Sig. $\left.=.002\right)$ y edad $\left(r_{s}=.21\right.$; Sig. $\left.=.002\right)$, e inteligencia espiritual con espiritualidad $\left(r_{s}=.57\right.$; Sig. <.001) y edad $\left(r_{s}=.15 ;\right.$ Sig. $\left.=.01\right)($ ver la tabla 4$)$.

Tabla 4

Correlación de ingresos económicos, espiritualidad y edad con experiencia paranormal, inteligencia espiritual y satisfacción existencial

\begin{tabular}{lccc}
\hline & Ingresos $^{(1)}$ & Espiritualidad $^{(2)}$ & Edad \\
\hline Index Psi & $-.13^{*}$ & $.46^{* * *}$ & .08 \\
Satisfacción existencial & $.14^{*}$ & $.20^{* * *}$ & $.21^{* *}$ \\
Inteligencia espiritual & -.10 & $.57^{* * *}$ & $.15^{* *}$ \\
\hline
\end{tabular}

${ }^{*} p<.05 ;{ }^{* *} p<.005 ;{ }^{* * *} p<.001$

(1) "Muy por debajo de lo aceptable para vivir" (1) a "Muy por encima de lo aceptable para vivir" (4).

(2) "No soy espiritual" (1) a "Soy extremadamente espiritual" (5).

Elaboración propia

Además, se reagrupó la variable religión en tres grupos: (1) "Religioso" (católico + judío), (2) “New Age/orientalismo" y (3) "Ateo/agnóstico (no creyente)". Luego se comparó las variables Index Psi, inteligencia espiritual y satisfacción existencial. Se encontró que aquellos que participan en New Age/orientalismo puntúan más alto en Index Psi $(p<.001)$ e inteligencia espiritual ( $p$ <.001) en comparación con los religiosos y ateos (ver la tabla 5 ).

Tabla 5

Comparación de religión con experiencia paranormal, inteligencia espiritual y satisfacción existencial

\begin{tabular}{|c|c|c|c|c|c|c|c|c|}
\hline \multirow[b]{2}{*}{ Variables } & \multicolumn{2}{|c|}{$\begin{array}{l}\text { Religioso } \\
(n=94)\end{array}$} & \multicolumn{2}{|c|}{$\begin{array}{c}\text { New Age/ } \\
\text { orientalismo } \\
\quad(n=85)\end{array}$} & \multicolumn{2}{|c|}{$\begin{array}{c}\text { Ateo/agnóstico } \\
\text { (no creyente) } \\
(n=21)\end{array}$} & \multirow[b]{2}{*}{$X^{2(1)}$} & \multirow[b]{2}{*}{$p$} \\
\hline & Media & DT & Media & DT & Media & DT & & \\
\hline Index Psi & 9,17 & 5,455 & 12,46 & 5,74 & 6,62 & 4,59 & 25,22 & $<.001$ \\
\hline $\begin{array}{l}\text { Inteligencia } \\
\text { espiritual }\end{array}$ & 46,70 & 18,692 & 58,07 & 18,52 & 35,38 & 14,39 & 29,42 & $<.001$ \\
\hline $\begin{array}{l}\text { Satisfacción } \\
\text { existencial }\end{array}$ & 88,57 & 17,817 & 87,75 & 20,77 & 84,95 & 13,82 & 1,36 & n. s. \\
\hline
\end{tabular}

(1) H de Kruskal-Wallis

Elaboración propia 


\section{DISCUSIÓN}

El objetivo general de este estudio fue evaluar la frecuencia de experiencias paranormales y su relación con la inteligencia espiritual y el nivel de satisfacción existencial; más específicamente, relacionar la frecuencia de experiencias paranormales con la inteligencia espiritual, relacionar la frecuencia de experiencias paranormales con la satisfacción existencial, relacionar la inteligencia espiritual con la satisfacción existencial, y analizar diferencias de género, ingresos económicos, espiritualidad y edad, religión y estado marital con respecto a la experiencia paranormal, la inteligencia espiritual y la satisfacción existencial.

Los resultados indican que un amplio porcentaje de los encuestados reportó al menos una experiencia ( $95 \%$ ), siendo la sensación de presencia (75\%) la más común, seguida de experiencias místicas, sueños premonitorios y percepción extrasensorial. Los resultados indican que la inteligencia espiritual es un fuerte predictor de la experiencia paranormal, y aunque la relación con la satisfacción existencial también quedó confirmada, esta significación solo fue positiva para experiencias específicas, tales como sueños premonitorios, experiencias extrasensoriales, sanar a distancia, y la capacidad de percibir y sensibilizarse por los valores, la claridad de sentimientos, la aceptación del prójimo y el otro, y el compromiso (sentir y compartir) como parte de la satisfacción existencial.

Las experiencias anómalas generan sentimiento espiritual y bienestar, lo que sugiere que diferentes tipos de experiencias pueden inducir efectos positivos, lejos de resultar perturbadoras. El hecho de que las experiencias paranormales produzcan reacciones positivas es importante para el campo de la psicología positiva, ya que demuestra que una experiencia paranormal puede contribuir a la capacidad del individuo para resolver cuestiones de significado y valor existencial, y sobre todo espiritual. Aquellas personas que han pasado por experiencias paranormales, sean o no de naturaleza perturbadora, podrían estar dotadas de estrategias más adaptativas frente a eventos anómalos o inexplicables, en principio. La inteligencia espiritual podría ser adaptativa para aquellos que buscan un significado a su vida espiritual. En efecto, aquellas personas que practican yoga, meditación, recuerdan sueños o tienen una vida mental más rica en fantasía comparten un contexto más amplio de significado frente a experiencias que acompañan su estilo de vida espiritual, es decir, una forma de "evidencia" que confirma el sentido y el valor de sus prácticas religiosas y/o espirituales. Estas experiencias pueden ser vistas como una señal que reafirma la congruencia entre sus creencias y sus prácticas espirituales.

En otras palabras, las experiencias paranormales podrían ser vistas como una prueba de existencia de una realidad trascendente, una confirmación de aquello intangible, o rara vez accesible u ominoso. La frecuencia con que estas experiencias se 
presentan flexibilizaría el sentido o propósito del mundo compartido con su creencia espiritual y sentido de la vida. Por ejemplo, una experiencia cercana a la muerte no solo puede resignificar positivamente el sentido de la vida (satisfacción existencial), sino también reforzar las teorías de una vida post mortem (inteligencia espiritual), y esto, a su vez, resignificar sus valores intra- e interpersonales y espirituales, o disminuir el miedo a la muerte (Greyson, 1992, 1993, 1996). Las experiencias de comunicación después de la muerte (por ejemplo, apariciones, oír voces y "sensación de presencia") pueden ser útiles para reforzar los lazos con las personas fallecidas en viudo(as) que han perdido a sus cónyuges o padres que han perdido hijos (Cooper, 2016; Steffen, Wilde y Cooper, 2017; Yoffe, 2012a, 2012b); las experiencias de sanación a distancia pueden revelar la capacidad del individuo para promover el bienestar y la salud emocional y espiritual de los otros (posiblemente mediatizado por la empatía, ver Parra, 2018).

Además, aquellos que descubren la habilidad de "salir de sus cuerpos" (EFC) tienen mayor control sobre futuras experiencias y mejores recursos cognitivos para preguntarse si las fronteras del yo van más allá de los límites establecidos (ver Alvarado, 2013; Craffert, 2015), y aquellas personas con experiencias psi espontáneas (por ejemplo, telepatía o premoniciones, ver Dossey, 2014) podrían resignificar los vínculos con sus seres queridos y la no linealidad del tiempo (Parra, 2015). Aquellos que dicen tener bajo control tales habilidades, por ejemplo, psíquicos, médiums y otros sensitivos también podrían resignificar un estilo de vida solidario con personas que buscan respuestas a sus aflicciones (Parra, 2011). Finalmente, las experiencias místicas también podrían ser útiles para aquellos involucrados en grupos religiosos o para sentirse acompañados positivamente por sus deidades, seres o guías espirituales (Cooper, 2016).

Aquellos que son más "inteligentes" espiritualmente podrían sencillamente ser más hábiles para afrontar experiencias anómalas, resignificarlas y evitar que estas resulten atemorizantes o carentes de control; pueden ser un recurso útil para tomar decisiones en términos del significado de tales experiencias o en el contexto de prácticas espirituales (como meditar u orar), hacer juicios sobre problemas existenciales o resolver problemas cotidianos. Por ejemplo, los estudios de Parra (2008) también sugieren que estas experiencias pueden estar asociadas a efectos secundarios mucho más positivos o saludables que perturbadores. Posiblemente, tener un sistema de creencias/experiencias paranormales pueda actuar como mecanismo protector, permitiendo aceptar y explicar tales experiencias, mientras que aquellos sin este recurso o con déficit cognitivo (por ejemplo, esquizoides) serían más vulnerables a la perturbación emocional que puede desencadenar un evento anómalo o inexplicable.

Otro resultado de este estudio es que - aunque no se encontró diferencia por género-se observó que aquellos con más experiencias tendían a mostrar mayor espiritualidad, y que la satisfacción existencial estaba relacionada con una situación económica 
mejorada. Tanto la satisfacción existencial como la inteligencia espiritual aumentaban también en relación con la edad; esto es, los adultos tendían a mostrar mayor satisfacción existencial e inteligencia emocional que los jóvenes. Además, aquellos que compartían creencias no convencionales (New Age/orientalismo) tendían a mostrar más inteligencia espiritual que los ateos, e incluso más que los practicantes de las religiones convencionales. Es posible que aquellos que siguen una vía de prácticas espirituales menos sujeta a dogmas y valores tradicionales tengan más adaptabilidad y capacidad para afrontar y resolver conflictos religiosos que aquellos sujetos a dogmas religiosos. Finalmente, la inteligencia espiritual parece menos comprometida con la vida marital en aquellos que conviven con sus cónyuges u otros familiares, para quienes el afrontamiento espiritual adhiere a un estilo de vida menos mundano que lo que exige la vida de compromisos sociales.

Futuros estudios deberían poner énfasis en muestras de individuos interesados en lo paranormal. Sin embargo, los datos revelan que este estudio presenta situaciones particulares en donde la inteligencia espiritual demuestra ser altamente adaptativa. Un vacío existencial puede resultar en una pérdida de significado de la vida o una frustración de la voluntad de significado. Tales crisis existenciales pueden ser el resultado del aislamiento, la inevitabilidad de la muerte (Yalom, 1980), el trauma y el dolor, un cambio cultural y la pérdida del empleo (Goddard, 2004). Niveles altamente desarrollados de pensamiento crítico existencial y producción de significado personal resultan particularmente valiosos, ya que facilitan tanto la llegada de la crisis como la creación de significado dentro de su contexto, evitando que muchas crisis potenciales emerjan.

\section{REFERENCIAS}

Alvarado, C. S. (2013). Psychic phenomena and the mind-body problem. Historical notes on a neglected conceptual tradition. Revista de Psiquiatría Clínica, 40(4), 157-161.

Baer, R. A. (2003). Mindfulness training as a clinical intervention: a conceptual and empirical review. Clinical Psychology: Science and Practice, 10(2), 125-143.

Barlow, D. H., y Durand, V. M. (2005). Abnormal psychology: an integrative approach (4. . ed.). Pacific Grove, CA: Brooks/Cole.

Bishop, S. R., Lau, M., Shapiro, S., Carlson, L., Anderson, N. D., y Carmody, J. (2004). Mindfulness: a proposed operational definition. Clinical Psychology: Science \& Practice, 11, 230-241.

Boado de Landaboure, N. B. (2001). Escala Existencial: manual introductorio. Buenos Aires: Dunken. 
Cardeña, E., Krippner, S., y Lynn, S. J. (Eds.). (2014). Varieties of anomalous experience: examining the scientific evidence (2. ${ }^{\text {a }}$ ed.). Washington, DC: American Psychological Association.

Cooper, C. E. (2016). The therapeutic nature of anomalous events. An union of positive psychology and parapsychology. En M. D. Smith y P. Worth (Eds.), $2^{\text {nd }}$ Applied Positive Psychology Symposium. Proceedings of Presented Papers (pp. 98-107). Buckinghamshire: Bucks New University.

Craffert, P. (2015). Do out-of-body and near-death experiences point towards the reality of nonlocal consciousness? A critical evaluation. Journal for Transdisciplinary Research in Southern Africa, 11(1), 1-20.

Dossey, L. (2014). El poder de las premoniciones. Conocer el futuro puede cambiar nuestra vida. Lérida: Milenio.

Emmons, R. A. (1999). The psychology of ultimate concerns: motivation and spirituality in personality. Nueva York, NY: Guilford.

Emmons, R. A. (2000a). Is spirituality an intelligence? Motivation, cognition, and the psychology of ultimate concern. International Journal for the Psychology of Religion, 10, 3-26.

Emmons, R. A. (2000b). Spirituality and intelligence: problems and prospects. The International Journal for the Psychology of Religion, 10, 57-64.

Goddard, N. C. (2004). Metamorphosis: spiritual transformation as response to suffering and trauma. Dissertation Abstracts International: Section B: The Sciences and Engineering, 65, 156

Gómez Montanelli, D., y Parra, A. (2004). A clinical approach to the emotional processing of anomalous/paranormal experiences in group therapy. Journal of the Society for Psychical Research, 68.3(876), 129-142.

Gómez Montanelli, D., y Parra, A. (2005). ¿Las experiencias paranormales son psicológicamente perturbadoras? Una encuesta comparando estudiantes universitarios y aficionados a temas paranormales. Revista Interamericana de Psicología, 39(2), 285-294.

Greyson, B. (1992). Reduced death threat in near-death experiencers. Death Studies, 16, 523-536.

Greyson, B. (1993). Near-death experiences and antisuicidal attitudes. Omega, 26(2), 81-89. 
Greyson, B. (1996). The near death experience as a transpersonal crisis. En B. W. Scotton, J. R. Battista y A. Chinen (Eds.), Textbook of transpersonal psychiatry and psychology (pp. 302-315). Nueva York, NY: Basic Books.

Holmes, D. S., Solomon, S., Cappo, B. M., y Greenberg, J. L. (1983). Effects of transcendental meditation versus resting on physiological and subjective arousal. Journal of Personality and Social Psychology, 44, 1245-1252.

Hölzel, B. K., Lazar, S. W., Gard, T., Schuman-Olivier, Z., Vago, D. R., y Ott, U. (2011). How does mindfulness meditation work? Proposing mechanisms of action from a conceptual and neural perspective. Perspectives on Psychological Science, 6(6), 537-559.

Kennedy, J. E., y Kanthamani, H. (1995). An exploratory study of the effects of paranormal and spiritual experiences on peoples' lives and well-being. Journal of the American Society for Psychical Research, 89, 249-265.

Kennedy, J. E., Kanthamani, H., y Palmer, J. (1994). Psychic and spiritual experiences, health, well-being, and meaning in life. Journal of Parapsychology, 58, 353-383.

King, D. B., y DeCicco, T. L. (2009). A viable model and self-report measure of spiritual intelligence. International Journal of Transpersonal Studies, 28(1), 68-85.

King, D. B., Mara, C., y DeCicco, T. L. (2012). Connecting the spiritual and emotional intelligences: confirming an intelligence criterion and assessing the role of empathy. International Journal of Transpersonal Studies, 31, 11-20.

Längle, A. (2004). Psicoterapia analítico-existencial de los trastornos depresivos. Revista Chilena de Neuro-Psiquiatría, 42(3), 195-206.

Lehmann, D., Faber, P. L., Achermann, P., Jeanmonod, D., y Gianotti, L. R. R. (2001). Brain sources of EEG gamma frequency during volitionally mediation-induced altered states of consciousness, and experience of the self. Psychiatry Research: Neuroimaging, 108(2), 111-121. https://doi.org/10.1016/S0925-4927(01)00116-0

Love, P. G. (2002). Comparing spiritual development and cognitive development. Journal of College Student Development, 43(3), 357-373.

Mascaro, N., y Rosen, D. H. (2005). Existential meaning's role in the enhancement of hope and prevention of depressive symptoms. Journal of Personality, 73, 985-1014.

Noble, K. D. (2000). Spiritual intelligence: a new frame of mind. Spirituality and Giftedness, 9, 1-29.

Paloutzian, R. F., y Ellison, C. W. (1982). Loneliness, spiritual well-being and the quality of life. En L. A. Peplau y D. Perlman (Eds), Loneliness: a sourcebook of current theory, research and therapy (pp. 224-237). Nueva York, NY: Wiley. 
Parra, A. (2008). Efectos de las experiencias espirituales/paranormales en la vida de las personas y su bienestar psicológico. Revista Argentina de Clínica Psicológica, 17(3), 233-244.

Parra, A. (2011). ¿Qué es... sensibilidad psíquica? Buenos Aires: Longseller.

Parra, A. (2015). Personality traits associated with premonition experience: neuroticism, extraversion, empathy, and schizotypy. Journal of the Society for Psychical Research, 79.1(918), 1-10.

Parra, A. (2018). Empatía cognitiva y emocional en relación con cinco experiencias paranormales. Revista de Ciencias Sociales, 4(1), 95-106.

Parra, A., y Argibay, J. C. (2013a). A free-response ESP test in two hypnotic susceptibility groups: a pilot study. Australian Journal of Parapsychology, 13(1), 27-35.

Parra, A., y Argibay, J. C. (2013b). Anomalous remote diagnosis: mental and motor psi impressions under iconic representation of the person-target. Journal of Parapsychology, 77, 123-130.

Parra, A., y Corbetta, J. (2013). Experiencias paranormales y su relación con el sentido de la vida. Liberabit, 19(2), 251-258.

Piechowski, M. M. (2001). Childhood spirituality. Journal of Transpersonal Psychology, 33, 1-15.

Seligman, M. E. P., y Csikszentmihalyi, M. (2000). Positive psychology: an introduction. American Psychologist, 55, 5-14.

Sheldon, K. M., y King, L. (2001). Why positive psychology is necessary. American Psychologist, 56, 216-217.

Sin, N. L., y Lyubomirsky, S. (2009). Enhancing well-being and alleviating depressive symptoms with positive psychology interventions: A practice-friendly meta-analysis. Journal of Clinical Psychology, 65(5), 467-487.

Srivastava, P. S. (2014). Interaction effect of sex, achievement motivation, and emotional intelligence on spiritual intelligence of pupil-teacher. Scholarly Research Journals, 5-2(15), 2286-2306.

Steffen, E., Wilde, D., y Cooper, C. E. (2017). Affirming the positive in anomalous experiences: a challenge to dominant accounts of reality, life and death. En N. J. Brown, T. Lomas y F. J. Eiroa-Orosa (Eds.), The Routledge International Handbook of Critical Positive Psychology (pp. 227-244). Londres: Routledge.

Vaughan, F. (2002). What is spiritual intelligence? Journal of Humanistic Psychology, 42(2), 16-33. 
Wigglesworth, C. (2006). Why spiritual intelligence is essential to mature leadership. Integral Leadership Review, 6, 68-79.

Wolman, R. (2001). Thinking with your soul: spiritual intelligence and why it matters. Nueva York, NY: Harmony.

Yalom, I. (1980). Existential psychotherapy. Nueva York, NY: Basic Books.

Yoffe, L. (2012a). Beneficios de las prácticas religiosas/espirituales en el duelo. Avances en Psicología Latinoamericana, 20(1), 9-29.

Yoffe, L. (2012b). La religión y la espiritualidad en los duelos desde la visión de la psicología positiva. En M. M. Casullo (Ed.), Simposio de Psicología Positiva, 4, 83-116.

Zohar, D. (1997). Rewiring the corporate brain: using the new science to rethink. Nueva York, NY: Diane.

Zohar, D. (2000). SQ. Connecting with our spiritual intelligence. Londres: Bloomsbury. 